\title{
Características bioquímicas e hematológicas do pirarucu Arapaima gigas Schinz, 1822 (Arapaimidae) de cultivo semi-intensivo na Amazônia
}

Gustavo Valadares Fonseca DRUMOND ${ }^{1}$, Ana Paula de Almeida CAIXEIROํ, Marcos TAVARES-DIAS², Jaydione Luiz MARCON ${ }^{3}$, Elizabeth Gusmão AFFONSO 4

\section{RESUMO}

O presente estudo comparou os níveis plasmáticos de glicose, proteínas totais, uréia, triglicerídeos e colesterol, a contagem de eritrócitos, leucócitos e trombócitos entre alevinos e juvenis de Arapaima gigas (Osteoglociformes, Arapaimidae) de uma piscicultura semi-intensiva de Manaus, estado do Amazonas, Brasil. Os alevinos de A. gigas apresentaram níveis significativamente $(\mathrm{p}<0,05)$ menores de proteínas totais, triglicerídeos, uréia e Volume Corpuscular Médio (VCM) e maior concentração de glicose, colesterol e hemoglobina, contagem de eritrócitos, hematócrito e Concentração da Hemoglobina Corpuscular Média (CHCM) quando comparados aos juvenis. Porém, não houve diferença significativa ( $>0,05)$ no número de trombócitos e leucócitos totais entre alevinos e juvenis. Os juvenis de $A$. gigas apresentaram menor quantidade de linfócitos e maior quantidade de monócitos, neutrófilos e eosinófilos, quando comparados aos alevinos. Em estudos futuros, espécimes de $A$. gigas com outras idades também deverão ser comparados para melhor entendimento dos efeitos da idade neste peixe amazônico, pois estas informaçôes, além de possibilitarem uma melhor compreensão da fisiologia desta espécie, poderão auxiliar no estabelecimento de estratégias para seu manejo.

PALAVRAS-CHAVE: Cultivo, Eritrócitos, Idade, Leucócitos, Sangue.

\section{Biochemical and hematological characteristics of the pirarucu Arapaima gigas Schinz, 1822 (Arapaimidae) from semi-intensive culture in the Amazon}

\section{ABSTRACT}

The present study compared the biochemical and hematological parameters of fingerlings and juveniles Arapaima gigas (Osteoglociformes, Arapaimidae) from a semi-intensive fish farming in Manaus, Amazon State, Brazil. Fingerlings A. gigas had lower levels of total protein, triglycerides, urea and mean cell volume $(\mathrm{MCV})$, but higher $(\mathrm{p}<0.05)$ glucose, cholesterol and hemoglobin concentration, red blood cell counts, hematocrit and cell hemoglobin concentration (MCHC) when compared to juveniles. However, there was not significant difference $(\mathrm{p}>0.05)$ in the total thrombocytes and total white blood cell counts between fingerlings and juveniles. Juveniles $A$. gigas presented lower lymphocytes number and higher monocytes, neutrophils and eosinophils number when compared to the fingerlings. In further studies, $A$. gigas specimens with other ages should also be compared for a better understanding of the ontogenic development in this Amazon fish, because this information besides increase the knowledge of the physiology of this species, can aid in the establishment of strategies for its adequate handling. KEYWORDS: Culture, Erythrocytes, Age, Leukocytes, Blood.

\footnotetext{
${ }^{1}$ Centro Universitário do Leste de Minas Gerais. Avenida Bárbara Heliodora, 725, Bom Retiro, 35160215, Ipatinga, MG.

2 Embrapa Amapá. Rodovia Juscelino Kubitschek, km 5, № 2600, Caixa Postal: 10, 68903-419, Macapá, AP, Brasil. Fone: (96) 4009-9550 e Fone/Fax (96) 4009-9501. e-mails: marcostavares@cpafap.embrapa.br; mtavaresdias@pq.cnpq.br.

${ }^{3}$ Departamento de Ciências Fisiológicas, Instituto de Ciências Biológicas, Universidade Federal do Amazonas (UFAM). Avenida. General Rodrigo Octávio Jordão Ramos, 3000, Coroado I, 69077-000, Manaus, AM

${ }^{4}$ Coordenação de Pesquisa em Aquicultura (CPAQ), Instituto Nacional de Pesquisas da Amazônia (INPA). Avenida André Araújo 2936, 69.060-001, Manaus, AM.
} 


\section{INTRODUÇÃO}

Dentre as espécies de peixes amazônicos de importância econômica, encontra-se o Arapaima gigas (pirarucu), pertencente à ordem Osteoglossiformes, Arapaimidae. Este peixe carnívoro, que é uma das cinco espécies mais cultivadas e comercializadas na Amazônia, tem características propícias para cultivo, tais como: a alta taxa de crescimento (até $10 \mathrm{~kg}$ no primeiro ano), elevada rusticidade ao manuseio (Crescêncio 2001; Cavero et al. 2003a,b; Cavero et al. 2004; Ono et al. 2004; Souza et al. 2006), adaptabilidade à alimentação artificial (Crescêncio 2001; Cavero et al. 2003a,b; Tavares-Dias et al. 2007) e elevado aproveitamento da carcaça (51-57\%) (Crescêncio 2001; Souza et al. 2006). O A. gigas é uma espécie de respiração aérea obrigatória, sendo que tal característica facilita sua criação (Crescêncio 2001; Cavero et al. 2003a,b; Ono et al. 2004) em elevadas densidades de estocagem (Scorvo Filho et al. 2004), bem como em baixas concentraçóes de oxigênio dissolvido na água.

Devido à reduçáo das populaçôes naturais pela pesca extrativista e predatória (Tavares-Dias et al. 2000) há a necessidade de se conhecer as características biológicas das diferentes espécies, principalmente referentes aos parâmetros fisiológicos (Ranzani-Paiva e Eiras, 1992; Kavamoto et al. 1983; Tavares-Dias e Moraes, 2004). Porém, pouco se conhece sobre as características hematológicas do $A$. gigas, pois até o presente momento foram estudados somente os parâmetros bioquímicos e hematológicos para esta espécie em tanquesrede (Tavares-Dias et al. 2007) e quando suplementados com vitaminas C e E (Menezes et al. 2006; Andrade et al. 2007), sendo que estes estudos são ainda incompletos para as várias fases de desenvolvimento ontogênico.

Alguns estudos brasileiros citam que a idade dos peixes é um dos fatores que normalmente interfere nos parâmetros sanguíneos (Ranzani-Paiva \& Eiras 1992; Tavares-Dias e Moraes 2004; Tavares-Dias et al. 2008), mas nestes, os efeitos do desenvolvimento ontogênico não tem sido investigado. Porém, em espécies de clima temperado estes estudos referemse, em geral, a comparaçóes entre peixes jovens e sexualmente maduros e variação sazonal do ciclo reprodutivo (Hrubec et al. 2001), de forma que poucos trabalhos sobre estas alteraçóes foram sistematicamente documentados. Experimentos com carpas Cyprinus carpio Linnaeus, 1758 (Cyprinidae) demonstraram que o hematócrito e a hemoglobina variam em relação à idade dos indivíduos (Svetina et al. 2002). Em esturjōes Acipenser percicus Borodin, 1897 e Huso huso Linnaeus, 1758 (Acipenseridae), com o avanço da idade ocorreu aumento nos parâmetros hematológicos (Bahmani et al. 2001). Hrubec et al. (2001) relataram que no híbrido Morone chrysops x $M$. saxatilis, as alteraçōes sanguíneas relacionadas à idade foram similares àquelas conhecidas para trutas Oncorhynchus mykiss Walbaum, 1792 (Salmonidae), bem como para mamíferos.

Os parâmetros bioquímicos metabólicos e hematológicos são ferramentas úteis para se determinar as características sanguíneas dos peixes (Centeno et al. 2007; Tavares-Dias et al. 2007; Tavares-Dias e Moraes 2007), pois fornecem informaçôes relevantes sobre as condiçôes fisiológicas e a saúde do indivíduo ou de sua população, as quais são extremamente importantes em peixes cativos, para seu manejo e manutençáo. Os valores que correspondam ao estado saudável dos peixes podem apontar importantes informaçóes para diagnóstico e prognóstico das condiçôes mórbidas de indivíduo ou de sua população, ajudando na identificaçáa de estresse e enfermidades (Tavares-Dias e Moraes, 2004; Tavares-Dias et al. 2007; Tavares-Dias e Moraes 2007; Davis et al. 2008; Tavares-Dias et al. 2008). Os trombócitos são células multifuncionais que estão envolvidas no processo de coagulaçáo sanguínea e na defesa orgânica nos peixes, pois embora não sejam consideradas de linhagem leucocitária, também realizam fagocitose (Tavares-Dias e Moraes 2004; Tavares-Dias et al. 2007). Os leucócitos como são as células mais importantes para a defesa (Tavares-Dias et al. 2008; Davis et al. 2008) podem ser entáo usados para avaliar o sistema imunológico dos peixes (Tavares-Dias et al. 2007; Davis et al. 2008; Tavares-Dias et al. 2008).

Tendo em vista que a elucidação da relação entre parâmetros sanguíneos e desenvolvimento ontogênico poderá se tornar uma importante ferramenta para a patologia clínica (Hrubec et al. 2001), o presente trabalho teve como objetivo comparar os valores bioquímicos, eritrograma, trombograma e leucograma de alevinos e juvenis de $A$. gigas provenientes de sistema de cultivo semi-intensivo na Amazônia central.

\section{MATERIAL E MÉTODOS}

\section{ANIMAIS E CONDIÇÕES DE CULTIVO}

Vinte e quatros espécimes de Arapaima gigas, sendo quinze alevinos $(25,9 \pm 2,7 \mathrm{~g}$ e $16,1 \pm 0,6 \mathrm{~cm})$ e nove juvenis $(2.350,0 \pm 757,1 \mathrm{~g} \mathrm{e} 64,2 \pm 6,7 \mathrm{~cm})$, foram coletados em uma piscicultura semi-intensiva no município de Manaus (Estado do Amazonas, Brasil), e usados para exames os bioquímicos e hematológicos. A manutenção dos alevinos foi realizada em viveiro escavado $\left(470 \mathrm{~m}^{2}\right)$, e em consórcio com tartarugas da Amazônia Podocnemis expansa Schweigger, 1812 (Pelomedusidae) e tilápia do Nilo Oreochromis niloticus Linnaeus, 1758 (Cichlidae). Todos os alevinos utilizados neste trabalho, obtidos com aproximadamente 30 dias de idade, foram oriundos da mesma desova, a qual ocorreu em condiçóes naturais. Os juvenis também foram mantidos em viveiro escavado $\left(80 \mathrm{~m}^{2}\right)$, sendo alimentados uma vez ao dia com pequenos peixes vivos ou mortos (tilápias e acarás). 
$\mathrm{Na}$ coleta dos alevinos e na outra coleta dos juvenis, o potencial hidrogeniônico $(\mathrm{pH})$, temperatura, condutividade elétrica e oxigênio dissolvido foram medidos com equipamentos digitais, para estas finalidades.

\section{PROCEDIMENTOS DE COLETA DO SANGUE E ANÁLISES}

As amostras de sangue de cada peixe foram colhidas por punçáo do vaso caudal, com auxílio de seringas de $3 \mathrm{~mL}$ (juvenis) e $1 \mathrm{~mL}$ (alevinos) contendo EDTA (10\%). Essas amostras de sangue foram usadas para realizar a contagem de eritrócitos em câmara de Neubauer, a determinação do hematócrito pelo método do microhematócrito e da concentração de hemoglobina pelo método da oxihemoglobina. A partir desses resultados foram calculados, segundo Wintrobe (1934), o volume corpuscular médio (VCM) e a concentração de hemoglobina corpuscular média (CHCM).

O sangue também foi usado para confecção de extensões sanguíneas coradas com uma combinação de May GrünwaldGiemsa-Wright (Tavares-Dias e Moraes 2003). As extensôes sanguíneas foram usadas para contagem diferencial de leucócitos, onde foram contadas 200 células em cada extensão sanguínea, estabelecendo-se o percentual de cada componente celular de interesse, identificados como: linfócitos, monócitos, neutrófilos e eosinófilos, seguindo as recomendaçôes de Tavares-Dias et al. (2007). Estas extensóes sanguíneas foram usadas também para a determinação do número de trombócitos e leucócitos totais (Tavares-Dias e Moraes, 2006).

O restante de cada amostra de sangue foi centrifugado, durante 5 minutos a $750 \mathrm{G}$, para obtenção do plasma, usado para determinaçấo da concentraçáo da glicose pelo método da glicose-oxidase. Os níveis plasmáticos de proteínas totais, uréia, triglicerídeos e colesterol foram determinados usando um kit comercial (Doles, GO, Brasil).

\section{ANÁLISE ESTATÍSTICA}

Para comparação das variáveis obtidas entre alevinos e juvenis foi usado o teste $t$ de Student. Em todas as análises realizadas, assumiu-se um intervalo de confiança de $95 \%$ $(p<0,05)$.

\section{RESULTADOS \& DISCUSSÕES}

As características físico-químicas da água dos viveiros de A. gigas, demonstradas na Tabela 1 , mostra que os valores observados estão dentro dos limites sugeridos por Crescêncio (2001) como ideal para a criação de pirarucu na Amazônia. Porém, os valores da temperatura e dos níveis de oxigênio dissolvido foram maiores no viveiro de alevinos, quando comparado ao viveiro de juvenis. Os valores de temperatura foram próximos aos relatados por Tavares-Dias et al. (2007), para pirarucus criados em tanques-rede, enquanto os de oxigênio dissolvido foram menores que os valores encontrados
Tabela 1 - Características físico-químicas da água dos viveiros de terra dos alevinos e os juvenis de $A$. gigas provenientes de cultivo semi-intensivo na Amazônia central.

\begin{tabular}{lcccc}
\hline $\begin{array}{l}\text { Parâmetros } \\
\text { Viveiros }\end{array}$ & $\begin{array}{c}\text { Temperatura } \\
\left({ }^{\circ} \mathrm{C}\right)\end{array}$ & $\begin{array}{c}\text { Oxigênio } \\
\text { Dissolvido } \\
(\mathrm{mg} / \mathrm{L})\end{array}$ & $\mathrm{pH}$ & $\begin{array}{c}\text { Condutividade } \\
(\mu \mathrm{S} / \mathrm{cm})\end{array}$ \\
\hline Alevinos & 29,1 & 4,0 & 6,9 & 76,6 \\
Juvenis & 26,4 & 2,2 & 6,8 & 97,1 \\
\hline
\end{tabular}

por aqueles autores. Contudo, esses menores índices de oxigênio dissolvido não são prejudiciais para a criação de pirarucus, principalmente para juvenis, que durante o seu desenvolvimento passam a apresentar respiração aérea, devido a uma modificação em sua bexiga natatória (Brauner et al. 2004). Podemos assumir assim, que no presente estudo os parâmetros físico-químicos da água dos viveiros não influenciaram nos parâmetros bioquímicos e hematológicos de pirarucus.

A avaliação dos parâmetros sanguíneos possibilita uma rápida detecção do estresse que o ambiente de cultivo pode impor aos peixes (Tavares-Dias et al. 2003; Tavares-Dias et al. 2007; Tavares-Dias et al. 2008). Além disso, estas informaçôes podem ser utilizadas pela inspeção veterinária para avaliar e controlar o estado fisiológico dos peixes, padronizando condiçôes ideais para o cultivo de cada espécie (Svetina et al. 2002).

Em relação aos parâmetros sanguíneos, foi observado que os alevinos de pirarucu possuem níveis significativamente $(\mathrm{p}<0,05)$ menores de proteínas totais, triglicerídeos, uréia e VCM, mas uma maior concentraçâo de glicose e colesterol, contagem de eritrócitos, hemoglobina, hematócrito e $\mathrm{CHCM}$, quando comparados aos juvenis (Tabela 2). Porém, Centeno et al. (2007) encontraram aumento para o hematócrito e concentração de hemoglobina quando compararam alevinos e juvenis de tambaquis Colossoma macropomum Cuvier, 1818 (Characidae). Em esturjóes A. percicus e H. huso, o hematócrito aumentou com a idade dos peixes (Bahmani et al. 2001). Em híbrido de M. chrysops x M. saxatilis, foi relatado aumento dos níveis de proteínas totais, hematócrito, hemoglobina, VCM e número de eritrócitos com o aumento da idade, mas redução dos níveis de glicose e colesterol (Hrubec et al. 2001). Em carpas C. carpio o hematócrito e hemoglobina aumentaram durante o primeiro ano de vida (Svetina et al. 2002), quando o metabolismo dos peixes é maior, devido ao seu crescimento. Portanto, alteraçôes nos valores desses parâmetros sanguíneos podem ser esperadas devido ao crescimento dos tecidos hematopoiéticos (rim e baço), os quais continuam a se desenvolver nos peixes jovens (Hrubec et al. 2001).

No sangue de alevinos e juvenis de $A$. gigas foram encontrados trombócitos, linfócitos, monócitos, neutrófilos e eosinófilos com características morfológicas similares aquelas 
Tabela 2 - Valores médios \pm desvio padrão dos parâmetros bioquímicos e eritrograma de alevinos e juvenis de $A$. gigas provenientes de cultivo semiintensivo na Amazônia central.

\begin{tabular}{lcc}
\hline Parâmetros & Alevinos $(\mathrm{n}=15)$ & Juvenis $(\mathrm{n}=9)$ \\
\hline Proteínal total $(\mathrm{g} / \mathrm{dL})$ & $1,99 \pm 0,30 \mathrm{~b}$ & $3,5 \pm 0,7 \mathrm{a}$ \\
Glicose $(\mathrm{mg} / \mathrm{dL})$ & $61,9 \pm 11,3^{\mathrm{a}}$ & $48,5 \pm 16,6 \mathrm{~b}$ \\
Triglicerídeos $(\mathrm{mg} / \mathrm{dL})$ & $73,5 \pm 31,3 \mathrm{~b}$ & $397,6 \pm 185,9 \mathrm{a}$ \\
Colesterol $(\mathrm{mg} / \mathrm{dL})$ & $280,2 \pm 46,4 \mathrm{a}$ & $255,8 \pm 32,5 \mathrm{a}$ \\
Uréia $(\mathrm{mg} / \mathrm{dL})$ & $1,1 \pm 0,3 \mathrm{~b}$ & $2,4 \pm 0,4 \mathrm{a}$ \\
Eritrócitos $\left(\mathrm{x} 10^{6} / \mu \mathrm{L}\right)$ & $2,150 \pm 0,313 \mathrm{a}$ & $1,508 \pm 0,132 \mathrm{~b}$ \\
Hemoglobina $(\mathrm{g} / \mathrm{dL})$ & $9,1 \pm 1,0 \mathrm{a}$ & $8,2 \pm 0,6 \mathrm{~b}$ \\
Hematócrito $(\%)$ & $33,8 \pm 2,8 \mathrm{a}$ & $28,6 \pm 3,0 \mathrm{~b}$ \\
VCM $(\mathrm{fL})$ & $159,8 \pm 21,3 \mathrm{~b}$ & $189,5 \pm 14,2 \mathrm{a}$ \\
CHCM $(\mathrm{g} / \mathrm{dL})$ & $27,1 \pm 3,3 \mathrm{a}$ & $29,2 \pm 4,4 \mathrm{a}$ \\
\hline
\end{tabular}

Letras diferentes em uma mesma linha indicam diferenças significativas $(p<0,05)$ pelo teste t.

descritas por Tavares-Dias et al. (2007), para juvenis de pirarucu cultivados em tanques-rede. Em alevinos e juvenis de A. gigas os linfócitos e neutrófilos $(97,1 \%)$ foram os leucócitos mais frequentes à semelhança do está relatado para A. gigas (Tavares-Dias et al. 2007), Piaractus mesopotamicus Holmberg 1887 (Characidae) (Tavares-Dias e Mataqueiro, 2004), A. percicus e H. huso (Bahmani et al. 2001), M. chrysops x M. saxatilis (Hrubec et al. 2001) e Leporinus macrocephalus Garavello \& Britski, 1988 (Anostomidae) (Tavares-Dias et al. 2008). Linfócitos são células envolvidas na produçâo de imunoglobulinas e modulação da defesa, enquanto os neutrófilos são os leucócitos fagocíticos primários, os quais proliferam na circulação em resposta a infecçôes, inflamação e estresse (Davis et al. 2008), e portanto, são os leucócitos mais importantes na defesa imunológica do pirarucu, uma vez que monócitos e eosinófilos constituem juntos somente 2,9\% dos leucócitos desta espécie de teleósteo.

O número de trombócitos e leucócitos totais de alevinos e juvenis de $A$. gigas foram significativamente $(\mathrm{p}>0,05)$ similares. Porém, peixes juvenis apresentaram menor quantidade de linfócitos e maior quantidade de monócitos, neutrófilos e eosinófilos quando comparados aos alevinos (Tabela 3). Por outro lado, em híbrido de $M$. chrysops x M. saxatilis, o número de leucócitos em peixes com idade entre e 6 e 9 anos foi maior, diminuindo até os 19 meses de idade, enquanto o número de linfócitos e de trombócitos aumentaram (Hrubec et al. 2001). Em esturjóes A. percicus e $H$. huso o percentual de linfócitos aumentou com a idade, enquanto o de neutrófilos diminuiu (Bahmani et al. 2001). Como nos peixes juvenis os tecidos hematopoiéticos continuam a se desenvolver, consequentemente, deve-se esperar um desenvolvimento do sistema imunológico até a fase adulta (Hrubec et al. 2001), e o incremento dos fatores não humorais (células) pode ser tanto quantitativo e como qualitativo.
Tabela 3 - Valores médios \pm desvio padrão do trombograma e leucograma de alevinos e juvenis $A$. gigas provenientes de cultivo semi-intensivo na Amazônia central.

\begin{tabular}{lcc}
\hline Parâmetros & Alevinos $(\mathrm{n}=15)$ & Juvenis $(\mathrm{n}=9)$ \\
\hline Trombócitos $(\mu \mathrm{L})$ & $17470 \pm 10402 \mathrm{a}$ & $12377 \pm 3991 \mathrm{a}$ \\
Leucócitos $(\mu \mathrm{L})$ & $29883 \pm 1.1287 \mathrm{a}$ & $25091 \pm 6121 \mathrm{a}$ \\
Linfócitos $(\mu \mathrm{L})$ & $27173 \pm 10.978 \mathrm{a}$ & $14065 \pm 4496 \mathrm{~b}$ \\
Linfócitos $(\%)$ & $89,9 \pm 6,3 \mathrm{a}$ & $55,6 \pm 9,9 \mathrm{~b}$ \\
Monócitos $(\mu \mathrm{L})$ & $440 \pm 300 \mathrm{~b}$ & $1192 \pm 718 \mathrm{a}$ \\
Monócitos $(\%)$ & $1,5 \pm 1,1 \mathrm{~b}$ & $4,6 \pm 2,3 \mathrm{a}$ \\
Neutrófilos $(\mu \mathrm{L})$ & $1911 \pm 1230 \mathrm{~b}$ & $8381 \pm 2530 \mathrm{a}$ \\
Neutrófilos $(\%)$ & $7,2 \pm 4,9 \mathrm{~b}$ & $34,2 \pm 9,6 \mathrm{a}$ \\
Eosinófilos $(\mu \mathrm{L})$ & $360 \pm 328 \mathrm{~b}$ & $1452 \pm 713 \mathrm{a}$ \\
Eosinófilos $(\%)$ & $1,4 \pm 1,6 \mathrm{~b}$ & $5,7 \pm 2,1 \mathrm{a}$ \\
\hline
\end{tabular}

Letras diferentes em uma mesma linha indicam diferenças significativas $(p<0,05)$, pelo teste t.

\section{CONCLUSÃO}

O presente estudo demonstrou que alguns parâmetros sanguíneos do $A$. gigas foram influenciados pela idade dos peixes. Porém, para maiores conclusóes sobre os efeitos do desenvolvimento ontogênico nestes parâmetros, estudos adicionais deverão ser conduzidos usando também espécimes jovens e adultos de pirarucus, além de alevinos e juvenis.

\section{AGRADECIMENTOS}

À Fundaçáo de Amparo à Pesquisa do Amazonas (Processos 2203/05 e 2204/05) e ao Conselho Nacional de Pesquisa e Desenvolvimento Tecnológico (Processo 35.0117/2005-5), pelo apoio financeiro e bolsa concedida, respectivamente. Ao CNPq pela bolsa (PQ) concedida a M. Tavares-Dias (Processo: 300472/2008-0).

\section{BIBLIOGRAFIA CITADA}

Andrade, J.I.A.; Ono, E.A.; Menezes, G.C.; Brasil, E.M.; Roubach, R.; Urbinati, E.C.; Tavares-Dias, M.; Marcon, J.L.; Affonso, E.G. 2007. Influence of diets supplemented with vitamin C and E on pirarucu (Arapaima gigas) blood parameters. Comparative Biochemistry and Physiology Part A, 146: 576-580.

Bahmani, M.; Kazemi, R.; Donskaya, P. 2001. A comparative study of some hematological features in young reared sturgeons (Acipenser persicus and Huso huso). Fish Physiology and Biochemistry, 24: 135-140.

Brauner, C.J.; Matey, V.; Wilson, J.M.; Bernier, N.J.; Val, A. L. 2004. Transition in organ function during the evolution of airbreathing: insights from Arapaima gigas an obligate air-breathing teleost from the Amazon. Journal of Experimental Biology, 207: 1433-1438.

Cavero, B.A.S.; Pereira-Filho, M.; Roubach, R.; Ituassú, D.R.; Gandra, A.L.; Crescêncio, R. 2003a. Stocking density effect growth homogeneity juvenile pirarucu in confined environments. 
Pesquisa Agropecuária Brasileira, 38: 103-107 (in Portuguese, with abstract in English).

Cavero, B.A.S.; Pereira-Filho, M.; Roubach, R.; Ituassu, D.R.; Gandra, A.L.; Crescêncio, R. 2003b. Stocking density effect on alimentary efficiency in juveniles pirarucu (Arapaima gigas) in a confined environment. Acta Amazonica, 33: 631-637 (In Portuguese, with abstract in English).

Cavero, B.A.S.; Pereira-Filho, M.; Bordinhon, A.M.; Fonseca, F.A.L.; Ituassú, D.R.; Roubach, R.; Ono, E.A. 2004. Tolerance of pirarucu juveniles to increasing ammonia concentration in a closed environment. Pesquisa Agropecuária Brasileira, 39: 513516 (In Portuguese, with abstract in English).

Centeno, L.; Silva-Acuña, R.; Barrios, R. Lugo, R.S.; Matute, C.; Pérez, J.L. 2007.Hematological characteristics of cachama (Colossoma macropomum) in three phases of the growth in Delta Amacuro, Venezuela. Zootecnia Tropical, 25: 237-243 (In Spanish, with abstract in English).

Crescêncio, R. 2001. Alimentary training of alevins pirarucu Arapaima gigas (Cuvier, 1829), using alimentary attractions. Dissertação de Mestrado, Instituto Nacional de Pesquisas da Amazônia/Fundaçâo Universidade do Amazonas, Manaus, Amazonas. 35pp. (In Portuguese).

Davis, A.K.; Maney, D.L.; Maerz, J.C. 2008. The use of leukocytes profiles to measure stress in vertebrates: a review for ecologists. Functional Ecology, 22: 760-772.

Hrubec, T.C.; Smith, S.A.; Robertson, J.L. 2001. Age-related changes in hematology and plasma chemistry values of hybrid striped bass (Morone chrysops x Morone saxatatilis). Veterinary Clinical Pathology, 30: 8-15.

Kavamoto, E.T.; Tokumaru, M.; Silva, R.A.P. de S.; Campos, B.E.S. 1983. Some blood observations of the "cascudo"m Plecostomus albopunctatus Regan (1908). Boletim do Instituto de Pesca, 10: 101-106 (In Portuguese, with abstract in English).

Menezes, G.C.; Tavares-Dias, M.; Ono, E.A., Andrade; J.I.A.; Brasil, E.M.; Roubach, R.; Urbinati, E.C.; Marcon, J.L.; Affonso, E.A. 2006. The influence of dietary vitamin $\mathrm{C}$ and $\mathrm{E}$ supplementation on the physiological response of pirarucu, Arapaima gigas, in net culture. Comparative Biochemistry and Physiology Part A, 145:274-279. Ono, E.A.; Halverson, M.R.; Kubitza, F. 2004. Pirarucu - the forgotten giant. Panorama da Aquicultura, 81:1425 (In Portuguese).

Scorvo Filho, J.D.; Rojas, N.E.T.; Silva, C.M.; Konoike, T. 2004. Rearing of Arapaima gigas (Teleostei, Osteoglossidae) in hothouse and closed water circulation system in State of São Paulo, Brazil. Boletim do Instituto de Pesca, 30: 161-170 (In Portuguese, with abstract in English).

Souza, R.T.Y.B.; Oliveira, S.R.; Ono, E.A.; Andrade, J.I.A.; Brasil, E.M.; Marcon, J.L.; Tavares-Dias, M.; Affonso, E.G. 2006. Physiological responses of pirarucu Arapaima gigas Cuvier, 1829 (Osteoglossidae) transported with different sodium chloride concentrations, p.1048-1053. (www.revistaaquatic. com/civa2006). Acesso: 20/05/09 (in Portuguese, with abstract in English).

Svetina, A.; Matasin, Z.; Tofant, A.; Vucemilo, M.; Fijan, N. 2002. Haematological and some blood chemical parameters of young carp till the age of three years. Acta Veterinaria Hungarica, 50: 459-467.

Tavares-Dias, M.; Martins, M.L.; Moraes, F.R. 2000. Haematological characteristics of Brazilizan teleosts. V Parameters of piauçu Leporinus macrocephalus Garavello \& Britski, 1988 (Anostomidae). Naturalia, 25: 39-52 (In Portuguese, with abstract in English)

Tavares-Dias, M.; Moraes, F. R. 2003. Haematological evaluation of Tilapia rendalli Boulenger, 1896 (Ostrichthyes: Cichlidae) captured in a fee fishing farm in Franca Sáo Paulo State, Brazil. Bioscience Journal, 19: 103-110 (In Portuguese, with abstract in English).

Tavares-Dias, M.; Schalch, S.H.C.; Moraes, F.R. 2003. Hematological characteristics of brazilian teleosts. VII. Parameters of seven species collected in Guariba, São Paulo State, Brazil. Boletim do Instituto de Pesca, 29: 109-115.

Tavares-Dias, M.; Mataqueiro, M.I. 2004. Hematological, biochemical and biometric characteristics of Piaractus mesopotamicus Holmberg, 1887 (Osteichthyes: Characidae), reared in captivity. Acta Scientiarum, 24: 157-162 (In Portuguese, with abstract in English).

Tavares-Dias, M.; Moraes, F.R. 2004. Hematology of teleosts fish. 1. ed.: Villimpress, Ribeirão Preto. 144 pp (In Portuguese).

Tavares-Dias, M; Moraes, F.R. 2006. Hematological parameters for the Brycon orbignyanus Valenciennes, 1850 (Osteichthyes, Characidae) intensively bred. Hidrobiologica, 16: 271-274.

Tavares-Dias, M.; Moraes, F.R. 2007. Haematological and biochemical reference intervals for farmed channel catfish. Journal of Fish Biology, 71: 383-388.

Tavares-Dias, M.; Barcellos, J. F. M.; Marcon, J.L; Menezes, G.C.; Ono, E.A.; Affonso, E.G. 2007. Hematological and biochemical parameters for the pirarucu Arapaima gigas Schinz, 1822 (Osteoglossiformes, Arapaimidae) in net cage culture. Electronic Journal of Ichthyology, 2: 61-68.

Tavares-Dias, M.; Affonso, E.G.; Oliveira, S.R.; Marcon, J.L.; Egami. M.I. 2008. Comparative study on hematological parameters of farmed matrinxã, Brycon amazonicus Spix and Agassiz, 1829 (Characidae: Bryconinae) with others Bryconinae species. Acta Amazonica, 38: 799-806.

Wintrobe, M.M. 1934. Variations on the size and haemoglobin content of erythrocytes in the blood of various vertebrates. Folia Haematologica, 51: 32-49.

Recebido em 18/02/2009

Aceito em 07/12/2009 